\title{
Is Preoperative Fibrinogen Associated with the Survival Prognosis of Gastric Cancer Patients? A Multi-centered, Propensity Score- Matched Retrospective Study
}

\author{
Lin-Yong Zhao ${ }^{1} \cdot$ Yong-Liang Zhao $^{2} \cdot$ Jun-Jiang Wang $^{3} \cdot$ Qi-Di Zhao $^{4} \cdot$ Wen-Qi Yi ${ }^{4}$. \\ Qing Yuan ${ }^{4}$ Xin-Zu Chen ${ }^{1} \cdot$ Yong $\mathrm{Li}^{3} \cdot \mathrm{Kun} \mathrm{Yang}^{1} \cdot \mathrm{Xiao}$-Long Chen ${ }^{1} \cdot$ Wei-Han Zhang ${ }^{1}$. \\ Kai Liu ${ }^{1}$ Hua-Yang Pang ${ }^{1}$ - Danil Galiullin ${ }^{1,5} \cdot$ Hui Wang ${ }^{1} \cdot$ Li-Fei Sun ${ }^{1} \cdot$ Xiao-Hai Song ${ }^{1}$. \\ Jia-Bin Zheng ${ }^{3}$ Xue-Qing Yao ${ }^{3}$. Zong-Guang Zhou ${ }^{1} \cdot$ Pei-Wu Yu ${ }^{2} \cdot$ Jian-Kun Hu${ }^{1}$
}

Published online: 21 October 2019

(C) The Author(s) 2019

\begin{abstract}
Background The prognostic significance of preoperative plasma fibrinogen in patients with operable gastric cancer remains under debate. This study aimed to elucidate the prognostic value of fibrinogen in gastric cancer patients underwent gastrectomy.

Methods A total of 4351 patients with gastric cancer collected from three comprehensive medical centers were retrospectively evaluated. Patients were categorized by minimum $P$ value using X-tile, while the baseline confounders for fibrinogen was balanced through propensity score matching (PSM). The relationships between fibrinogen and other clinicopathologic features were evaluated, and nomogram was constructed to assess its prognostic improvement compared with TNM staging system.

Results Fibrinogen was significantly correlated with macroscopic type, tumor differentiation, tumor size, and $\mathrm{T}$ and $\mathrm{N}$ stage. The factors, fibrinogen and $\mathrm{T}$ stage as well as $\mathrm{N}$ stage, were identified to be independent prognostic factors after PSM. Nomogram based on fibrinogen demonstrated a smaller Akaike information criterion (AIC) and a larger concordance index (C-index) than TNM staging system, illustrating that fibrinogen might be able to improve the prognostic accuracy.

Conclusions Preoperative plasma fibrinogen levels in gastric cancer patients were significantly correlated with tumor progression, which could be regarded as a reliable marker for survival prognostic prediction.
\end{abstract}

Pei-Wu Yu

yupeiwu01@sina.com

$\triangle$ Jian-Kun $\mathrm{Hu}$

hujkwch@126.com

1 Department of Gastrointestinal Surgery and Laboratory of Gastric Cancer, State Key Laboratory of Biotherapy, West China Hospital, Sichuan University, and Collaborative Innovation Center for Biotherapy, No. 37 Guo Xue Xiang Street, Chengdu, Sichuan Province, China

2 Department of General Surgery and Center of Minimal Invasive Gastrointestinal Surgery, Southwest Hospital, Third Military Medical University, Chongqing 400038, China
3 Department of General Surgery, Guangdong General Hospital, Guangdong Academy of Medical Sciences, Guangzhou, China

4 West China School of Medicine, Sichuan University, Chengdu, China

5 Department of Urology, Bashkir State Medical University, Ufa, Russian Federation 


\section{Introduction}

Being the secondary leading cause of cancer-related mortality in China, gastric cancer is one of the most common gastrointestinal malignances worldwide, despite that global incidence is declining [1]. Identification of prognostic factors for gastric cancer becomes increasingly crucial in predicting patients' survival. The hypercoagulability of blood has been demonstrated to be associated with malignancies $[2,3]$. As an important coagulation factor, plasma fibrinogen synthesized by liver, which can be converted to fibrin by activated thrombin, has been recently illustrated to participate in tumorigenesis, stroma formation, angiogenesis, and tumor metastasis [4-7]. Nevertheless, previous studies showed that fibrinogen could be regarded as an independent predictor [8-11], whereas some proposed that it was not related to the survival outcome of patients with malignancy [12[. Moreover, there has been no unanimous consensus reached on what clinical roles were played by fibrinogen so far, and the clinical significance of preoperative fibrinogen has not been well addressed for gastric cancer patients till now [8, 13-16].

It is highly necessary, therefore, to elucidate the prognostic significance of fibrinogen and to determine its potential clinical value in gastric cancer patients. We aimed to investigate the prognostic significance of preoperative plasma fibrinogen levels in gastric cancer patients enrolled in three medical centers.
Patients and methods

\section{Patients}

From January 2002 to June 2012, 4351 gastric cancer patients, who underwent gastrectomy in West China Hospital, Southwest Hospital, and Guangdong General Hospital, were analyzed in this study. Exclusion criteria for patients were as follows: (1) multiple stomach tumors; (2) palliative surgical operation; (3) death because of postoperative complications in hospital stay; (4) peritoneal dissemination or distant metastasis; (5) patients who were lost to follow-up; (6) incomplete medical records. Finally, 3072 candidates were totally included as shown in Fig. 1. Specifically, 2737 of them, who were enrolled in Southwest Hospital and West China Hospital, were randomized with a sample size ratio $2: 1$, into inner training cohort and inner validation cohort, while 335 candidates in Guangdong General Hospital were defined as an independent validation cohort.

\section{Definition of fib-high/low groups and baseline characteristics}

On the basis of optimal cutoff point, $3.20 \mathrm{~g} / \mathrm{L}$, determined by $\mathrm{X}$-tile, gastric cancer patients were categorized into Fibhigh group $(\geq 3.20 \mathrm{~g} / \mathrm{L})$ and Fib-low group $(<3.20 \mathrm{~g} / \mathrm{L})$. The clinicopathologic and demographic factors included

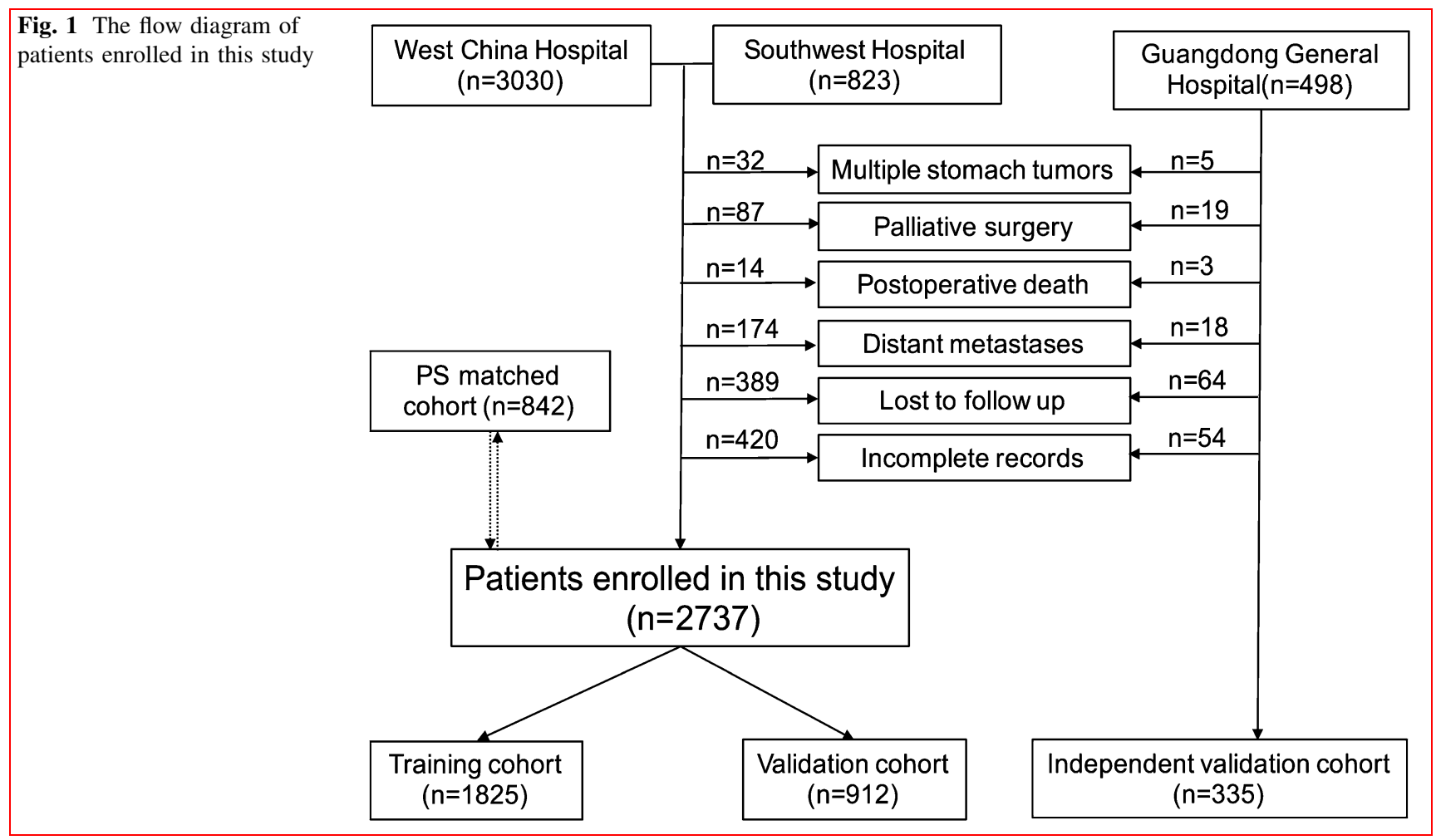


age, gender, extent of gastrectomy, surgical approach, tumor differentiation, tumor location, tumor size, macroscopic type, and pathological TNM stage[17].

\section{Follow-up and survival}

Patients were periodically followed up through telephone interviews, outpatient visits, and letters. The follow-up was arranged as bellows: every 3 months during the first 2 years after surgery, and then every 6 months in the following 3 years, and thereafter every 12 months till death. The survival time was calculated from the date of operation to the last follow-up time, June 2017, or date of death. Overall survival in this study was the primary endpoint. The changed telephone numbers or contact address and patients' refusal to the outpatients visit were the main cause to follow-up losses. Of the 4351 patients, 3898 (89.6\%) were followed up.

\section{Statistical analysis}

Optimal cutoff point of preoperative fibrinogen was selected from log-rank $\chi^{2}$ statistics by minimum $P$ value using the X-tile program [18]. Ranked and unordered categorical variables were assessed by Mann-Whitney U and Chi-square test, respectively, by SPSS (SPSS, version 23.0, Inc., Chicago, IL, USA). Cox's regression model was used to perform univariate and multivariate survival analyses. The log-rank test and Kaplan-Meier method were utilized to calculate cumulative survival rates.

To reduce the impact of potential confounding factors and effects of selection bias, such as baseline clinicopathologic factors or uneven patient distribution between the Fib-high and Fib-low groups, 1:1 propensity score matching was applied to adjust for gender, age, tumor size, macroscopic type, tumor differentiation, and $\mathrm{T}$ stage and $\mathrm{N}$
Table 1 Comparison and validation of different cutoff points for fibrinogen

\begin{tabular}{lllll}
\hline Authors (ref.) & Cutoff point (g/L) & AIC & $\begin{array}{l}\text { C- } \\
\text { index }\end{array}$ & $P$ \\
\hline Yu et al. [8] & $1.50 ; 4.00$ & 865.1 & 0.732 & $<0.05$ \\
Yu et al. [13] & 3.90 & 930.3 & 0.687 & $<0.01$ \\
Arigami et al. [14] & 3.05 & 942.2 & 0.661 & $<0.01$ \\
Lee et al. [15] & 4.00 & 886.9 & 0.722 & $<0.05$ \\
Yamashita et al.[16] & 3.10 & 858.4 & 0.737 & $<0.05$ \\
This study & 3.20 & 855.7 & 0.743 & $<0.01$ \\
\hline
\end{tabular}

AIC Akaike information criterion, ref. reference

stage. A 0.2-width caliper of the standard deviation of the logit was applied to match across the two groups [19]. The Regression Modeling Strategies package in R program was used to display nomogram and calibration curve, whereas Harrell Miscellaneous package was applied to compare the predictive ability of survival among different staging systems. Additionally, the prognostic accuracy and discriminatory ability of each system were evaluated by concordance index (C-index) and Akaike information criterion (AIC), respectively. A larger C-index means a more accurate prognostic prediction [20], but a larger AIC value corresponds to a model with a worse discriminatory ability for predicting outcome [21]. A $p$ value of $<0.05$ (two side) was regarded to be statistically significant.

\section{Results}

\section{Clinicopathologic characteristics and correlation analysis}

X-tile plots showed that the optimal cutoff point for fibrinogen was $3.20 \mathrm{~g} / \mathrm{L}$ in gastric cancer patients (Fig. 2),

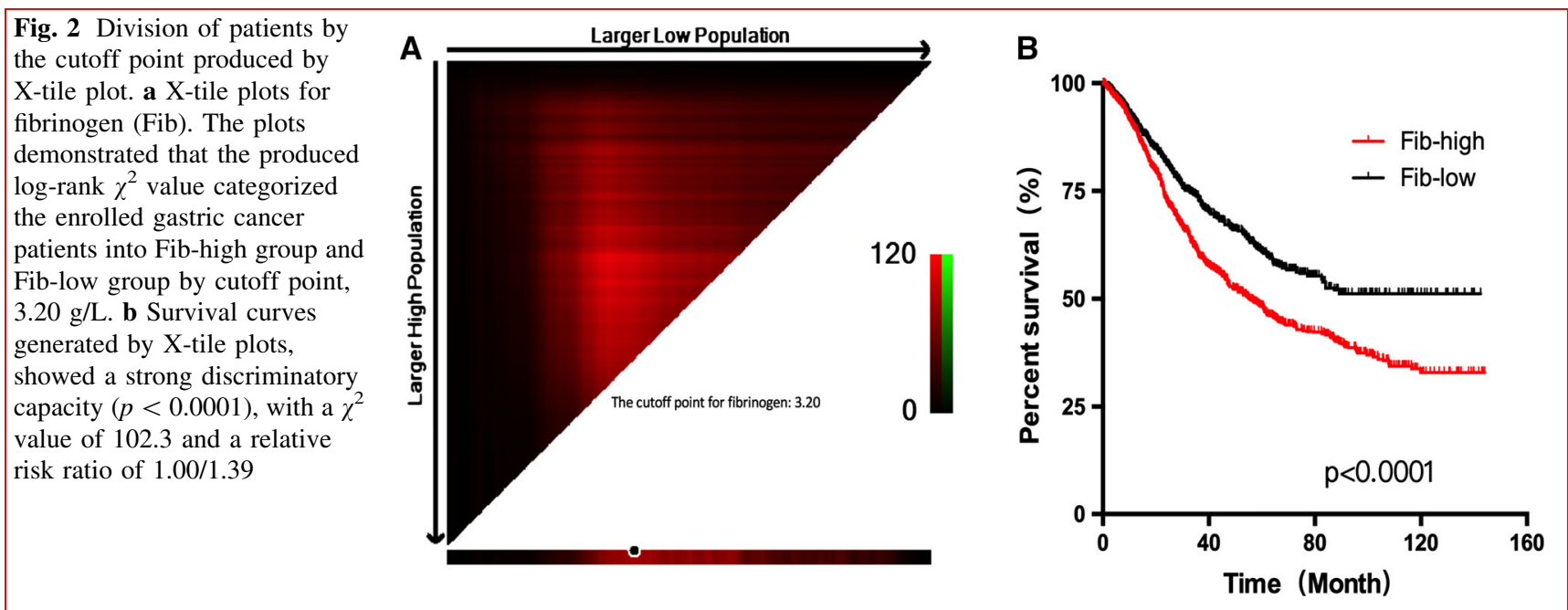


according to which patients were categorized into Fib-high group $(\geq 3.20 \mathrm{~g} / \mathrm{L})$ and Fib-low group $(<3.20 \mathrm{~g} / \mathrm{L})$. Moreover, various cutoff points proposed previously were validated using our sample to compare their prognostic prediction ability with the cutoff point produced in this study, as demonstrated in Table 1 . We found that

Table 2 Clinicopathologic factors of training, validation and propensity-score-matched cohorts stratified by preoperative plasma fibrinogen. $n(\%)$

\begin{tabular}{|c|c|c|c|c|c|c|c|c|c|}
\hline \multirow[t]{2}{*}{ Variables } & \multicolumn{3}{|c|}{ Training cohort $(n=1825)$} & \multicolumn{3}{|c|}{ Validation cohort $(n=912)$} & \multicolumn{3}{|c|}{$\begin{array}{l}\text { Propensity-score-matched cohort } \\
(n=842)\end{array}$} \\
\hline & $\begin{array}{l}\text { Fib-high } \\
(n=830)\end{array}$ & $\begin{array}{l}\text { Fib-low } \\
(n=995)\end{array}$ & $P$ value & $\begin{array}{l}\text { Fib-high } \\
(n=426)\end{array}$ & $\begin{array}{l}\text { Fib-low } \\
(n=486)\end{array}$ & $P$ value & $\begin{array}{l}\text { Fib-high } \\
(n=421)\end{array}$ & $\begin{array}{l}\text { Fib-low } \\
(n=421)\end{array}$ & $P$ value \\
\hline Gender & & & 0.110 & & & 0.074 & & & 0.327 \\
\hline Male & $602(72.5)$ & 687 (69.0) & & $298(70.0)$ & $366(75.3)$ & & $303(72.0)$ & $289(68.6)$ & \\
\hline Female & $228(27.5)$ & $308(31.0)$ & & $128(30.0)$ & $120(24.7)$ & & $118(28.0)$ & $132(31.4)$ & \\
\hline Age(years) & & & 0.059 & & & 0.102 & & & 0.262 \\
\hline$<65$ & $532(64.1)$ & $680(68.3)$ & & $278(65.3)$ & $342(70.4)$ & & $284(67.5)$ & $300(71.3)$ & \\
\hline$\geq 65$ & $298(35.9)$ & 315 (31.7) & & $148(34.7)$ & $144(29.6)$ & & $137(32.5)$ & $121(28.7)$ & \\
\hline Tumor location & & & 0.083 & & & 0.059 & & & 0.778 \\
\hline Upper third & $224(27.0)$ & $227(22.8)$ & & $70(16.4)$ & $74(11.1)$ & & $112(26.7)$ & $118(28.1)$ & \\
\hline Middle third & $120(14.4)$ & $138(13.9)$ & & $24(5.7)$ & $48(14.0)$ & & $60(14.2)$ & $57(13.5)$ & \\
\hline Lower third & $486(58.6)$ & $630(63.3)$ & & $332(77.9)$ & 364 (74.9) & & $249(59.1)$ & $246(58.4)$ & \\
\hline Tumor size $(\mathrm{cm})$ & & & 0.002 & & & 0.004 & & & 0.407 \\
\hline$<5$ & $454(54.7)$ & $589(59.2)$ & & $230(54.0)$ & $216(44.4)$ & & $232(55.2)$ & $219(52.1)$ & \\
\hline$\geq 5$ & $376(45.3)$ & $406(40.8)$ & & $196(46.0)$ & $270(55.6)$ & & $189(44.8)$ & $202(47.9)$ & \\
\hline $\begin{array}{l}\text { Tumor } \\
\text { differentiation }\end{array}$ & & & 0.001 & & & $<0.001$ & & & 0.194 \\
\hline Well/moderately & $266(32.1)$ & $251(25.2)$ & & $150(35.2)$ & $306(63.0)$ & & 75 (17.9) & $91(21.7)$ & \\
\hline $\begin{array}{l}\text { Poorly/ } \\
\text { undifferentiated }\end{array}$ & $564(67.9)$ & $744(74.8)$ & & $276(64.8)$ & $180(37.0)$ & & $346(82.1)$ & $330(78.3)$ & \\
\hline Macroscopic type & & & $<0.001$ & & & 0.007 & & & 0.200 \\
\hline Borrmann 0-II & $488(58.8)$ & $687(69.0)$ & & $256(60.1)$ & 334 (68.7) & & $254(60.3)$ & 273 (64.9) & \\
\hline Borrmann III-IV & $342(41.2)$ & $308(31.0)$ & & $170(39.9)$ & $152(31.3)$ & & $167(39.7)$ & $148(35.1)$ & \\
\hline Surgical approach & & & 0.384 & & & 0.367 & & & 0.108 \\
\hline Open & $578(69.6)$ & $674(67.7)$ & & $277(65.0)$ & $302(62.1)$ & & $270(64.1)$ & $292(69.4)$ & \\
\hline Laparoscopic & $252(30.4)$ & $321(32.3)$ & & $149(35.0)$ & $184(37.9)$ & & $151(35.9)$ & $129(30.6)$ & \\
\hline Gastrectomy extent & & & 0.194 & & & 0.117 & & & 0.341 \\
\hline Total & $302(36.4)$ & $401(40.3)$ & & $155(36.4)$ & 189 (38.9) & & $184(43.7)$ & $202(48.0)$ & \\
\hline Distal & $481(58.0)$ & $535(53.8)$ & & $248(58.2)$ & $269(55.3)$ & & $222(52.7)$ & $201(47.7)$ & \\
\hline Proximal & $47(5.7)$ & $59(5.9)$ & & $13(6.6)$ & $28(5.8)$ & & $15(3.6)$ & $18(4.3)$ & \\
\hline $\mathrm{T}$ stage & & & $<0.001$ & & & 0.001 & & & 0.160 \\
\hline $\mathrm{T} 1$ & $101(12.2)$ & $188(18.9)$ & & $64(15.0)$ & $103(21.2)$ & & 49 (11.6) & $57(13.5)$ & \\
\hline $\mathrm{T} 2$ & 105 (12.7) & $219(22.0)$ & & $76(17.8)$ & $113(23.3)$ & & $57(13.5)$ & $67(15.9)$ & \\
\hline $\mathrm{T} 3$ & $245(29.5)$ & $230(23.1)$ & & $112(26.3)$ & $126(26.0)$ & & $116(27.6)$ & 89 (21.1) & \\
\hline $\mathrm{T} 4$ & 379 (45.7) & 358 (36.0) & & $174(40.8)$ & 144(29.6) & & 199 (47.3) & $208(49.4)$ & \\
\hline $\mathrm{N}$ stage & & & 0.034 & & & 0.002 & & & 0.852 \\
\hline N0 & 143 (17.2) & $189(19.0)$ & & $80(18.8)$ & $123(25.3)$ & & $93(22.1)$ & 89 (21.1) & \\
\hline N1 & $250(30.1)$ & 337 (33.9) & & $102(23.9)$ & $141(29.0)$ & & $117(27.8)$ & $127(30.2)$ & \\
\hline $\mathrm{N} 2$ & 264 (31.8) & $256(25.7)$ & & 143 (33.6) & $113(23.3)$ & & $122(29.0)$ & $114(27.1)$ & \\
\hline N3 & $173(20.8)$ & $213(21.4)$ & & $101(23.7)$ & 109 (22.4) & & 89 (21.1) & 91 (21.6) & \\
\hline Chemotherapy & & & 0.353 & & & 0.006 & & & 0.251 \\
\hline Present & $693(83.5)$ & 814 (81.8) & & $326(76.5)$ & $408(84.0)$ & & 349 (82.9) & 335 (79.5) & \\
\hline Absent/unclear & $137(16.5)$ & $181(18.2)$ & & $100(23.5)$ & 78 (16.0) & & $72(17.1)$ & $86(20.5)$ & \\
\hline
\end{tabular}



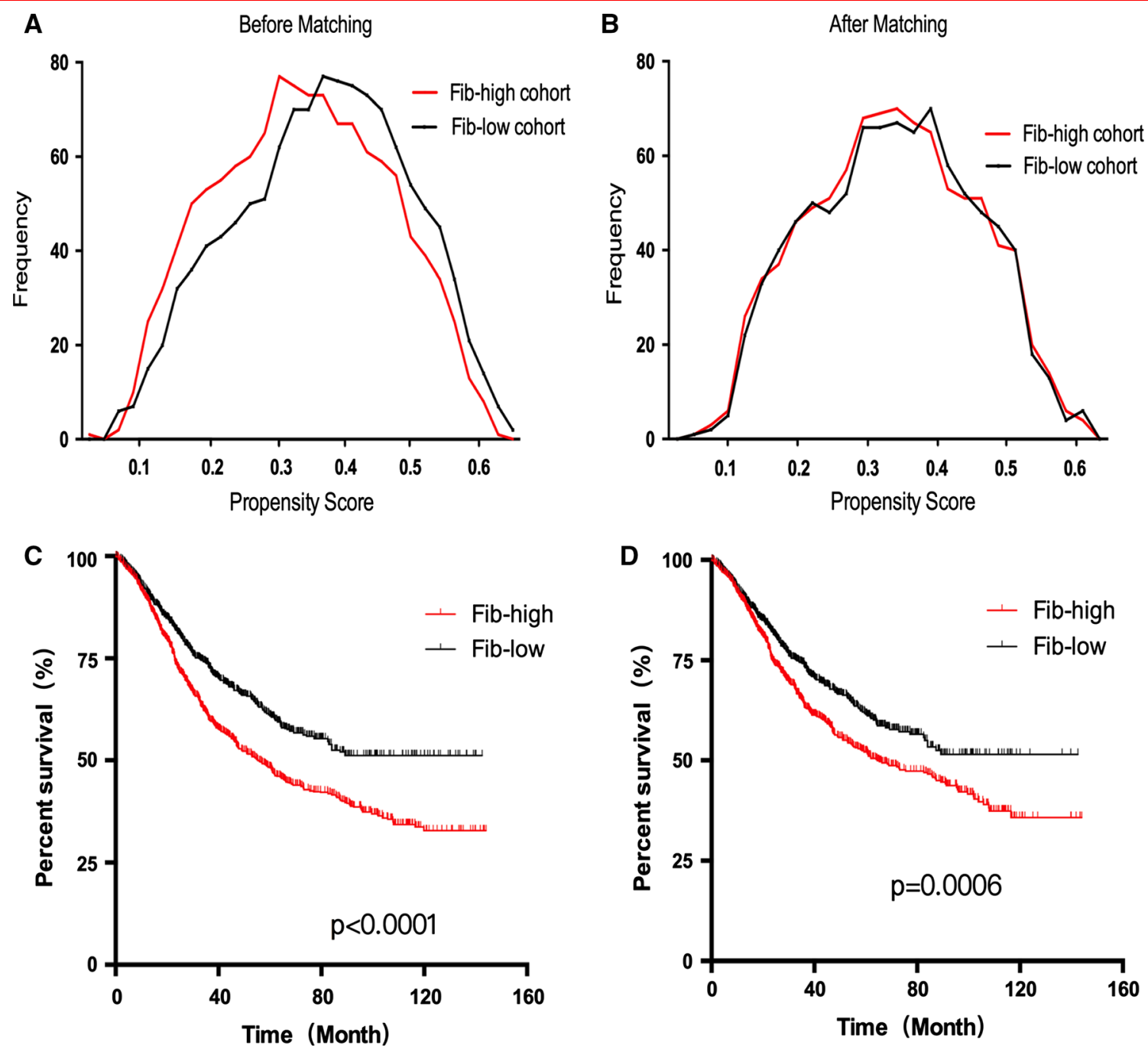

Fig. 3 Propensity score distributions of patients before matching (a) and after matching (b). Kaplan-Meier survival analyses of patients in the cohorts before matching (c) and after matching (d)

application of the cutoff point of $3.20 \mathrm{~g} / \mathrm{L}$ could produce a smaller AIC value as well as a larger C-index, as compared with other cutoff points reported previously (all $p<0.05$, Table 1).

Clinicopathologic features were compared between the Fib-high and Fib-low group in the training, validation, and propensity-score-matched cohort (Table 2). Fibrinogen was illustrated to be significantly associated with tumor differentiation $(p<0.05)$, tumor size $(p<0.05)$, macroscopic type $(p<0.05)$, T stage $(p<0.05)$, and $\mathrm{N}$ stage $(p<0.05)$ both in the training and in the validation cohort. Specifically, patients were more likely to be with macroscopic type III-IV, deep tumor invasion, and wide lymph nodes metastasis in Fib-high group than that in Fib-low group. Additionally, we found that poorly/undifferentiated histology was more prevalent in Fib-low group among training cohort but much more common in Fib-high group in validation cohort. Likewise, larger tumor size was associated with Fib-high in training cohort, while much more common in Fig-low in validation cohort. However, fibrinogen was not significantly associated with age $(p>0.05)$, gender $(p>0.05)$, tumor location $(p>0.05)$, gastrectomy extent $(p>0.05)$, and surgical approach $(p>0.05)$ in all of the cohorts.

\section{Prognostic significance of fibrinogen}

In order to balance the baseline and to reduce the potential confounding factor impact and selection bias effect, 1:1 propensity score matching was utilized to adjust for gender, age, tumor size, tumor differentiation, macroscopic type, and $\mathrm{T}$ stage and $\mathrm{N}$ stage between the Fib-high and Fib-low groups. Though an initial dissimilarity across the two groups was demonstrated in Fig. 3a according to 
Table 3 Cox regression analyses predicting overall survival in propensity-score-matched cohort

\begin{tabular}{|c|c|c|c|c|}
\hline \multirow[t]{2}{*}{ Variables } & \multicolumn{2}{|l|}{ Univariate analysis } & \multicolumn{2}{|l|}{ Multivariate analysis } \\
\hline & HR $(95 \%$ CI $)$ & $P$ value & HR $(95 \%$ CI $)$ & $P$ value \\
\hline \multicolumn{5}{|l|}{ Gender } \\
\hline Male & 1 & & 1 & \\
\hline Female & $0.843(0.612-0.904)$ & 0.035 & $0.749(0.632-1.022)$ & 0.381 \\
\hline \multicolumn{5}{|l|}{ Age (years) } \\
\hline$<65$ & 1 & & 1 & \\
\hline$\geq 65$ & $1.404(1.013-1.869)$ & 0.009 & $1.173(0.939-1.556)$ & 0.195 \\
\hline \multicolumn{5}{|l|}{ Fibrinogen } \\
\hline Fib-low & 1 & & 1 & \\
\hline Fib-high & $1.109(1.032-1.457)$ & 0.011 & $1.162(1.013-1.291)$ & 0.039 \\
\hline \multicolumn{5}{|l|}{ Tumor location } \\
\hline Upper & 1 & & & \\
\hline Lower/middle & $0.906(0.629-1.018)$ & 0.081 & - & - \\
\hline \multicolumn{5}{|l|}{ Tumor size } \\
\hline$<5 \mathrm{~cm}$ & 1 & & & \\
\hline$\geq 5 \mathrm{~cm}$ & $1.051(1.002-1.171)$ & 0.037 & $1.305(0.830-1.326)$ & 0.112 \\
\hline \multicolumn{5}{|l|}{ Tumor differentiation } \\
\hline Poorly/Undifferentiated & 1 & & 1 & \\
\hline Well/Moderately & $1.012(1.001-1.396)$ & 0.023 & $1.105(0.729-1.281)$ & 0.098 \\
\hline \multicolumn{5}{|l|}{ Macroscopic type } \\
\hline Borrmann III-IV & 1 & & 1 & \\
\hline Borrmann 0-II & $0.893(0.652-0.942)$ & 0.017 & $0.900(0.782-1.043)$ & 0.301 \\
\hline \multicolumn{5}{|l|}{ Surgical approach } \\
\hline Open & 1 & & - & - \\
\hline Laparoscopic & $0.989(0.678-1.201)$ & 0.078 & - & - \\
\hline \multicolumn{5}{|l|}{ Gastrectomy extent } \\
\hline Total & 1 & & - & - \\
\hline Distal & $0.877(0.679-1.103)$ & 0.069 & - & - \\
\hline Proximal & $1.125(0.897-1.524)$ & 0.103 & - & - \\
\hline \multicolumn{5}{|l|}{ T stage } \\
\hline $\mathrm{T} 1$ & 1 & & 1 & \\
\hline $\mathrm{T} 2$ & $1.739(1.019-2.284)$ & 0.005 & $1.374(1.026-1.872)$ & 0.026 \\
\hline $\mathrm{T} 3$ & $2.031(1.302-2.909)$ & 0.003 & $1.535(1.224-1.925)$ & 0.029 \\
\hline $\mathrm{T} 4$ & $2.894(1.804-4.538)$ & $<0.001$ & $2.601(1.852-3.968)$ & $<0.001$ \\
\hline \multicolumn{5}{|l|}{$\mathrm{N}$ stage } \\
\hline No & 1 & & 1 & \\
\hline N1 & $1.735(1.243-2.426)$ & 0.011 & $1.412(1.110-1.812)$ & 0.029 \\
\hline N2 & $2.214(1.382-2.996)$ & 0.009 & $1.687(1.223-2.048)$ & 0.019 \\
\hline N3 & $2.975(1.931-4.093)$ & $<0.001$ & $2.436(1.592-3.988)$ & 0.006 \\
\hline \multicolumn{5}{|l|}{ Chemotherapy } \\
\hline Present & 1 & & 1 & \\
\hline Absent/unclear & $1.156(1.004-1.921)$ & 0.035 & $1.043(0.831-1.721)$ & 0.191 \\
\hline
\end{tabular}

$H R$ hazard ratio, $C I$ confidence interval, - not enter the regression model

propensity score distributions before match, it was quite homogeneous after matching (Fig. 3b), illustrating the adequacy of balance achieved by the propensity score model, and all of the clinicopathologic features were found to be balanced (all $p>0.05$ ) between the two groups in Table 2. Moreover, patients with Fib-low were illustrated 


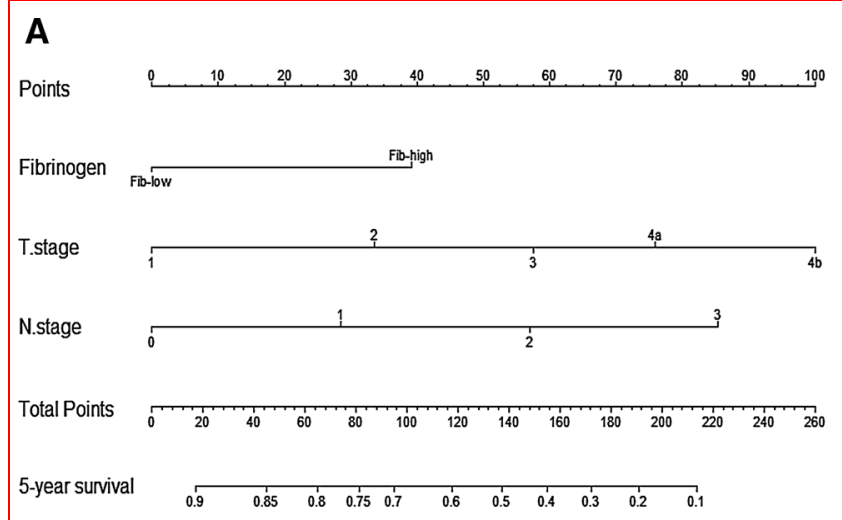

B

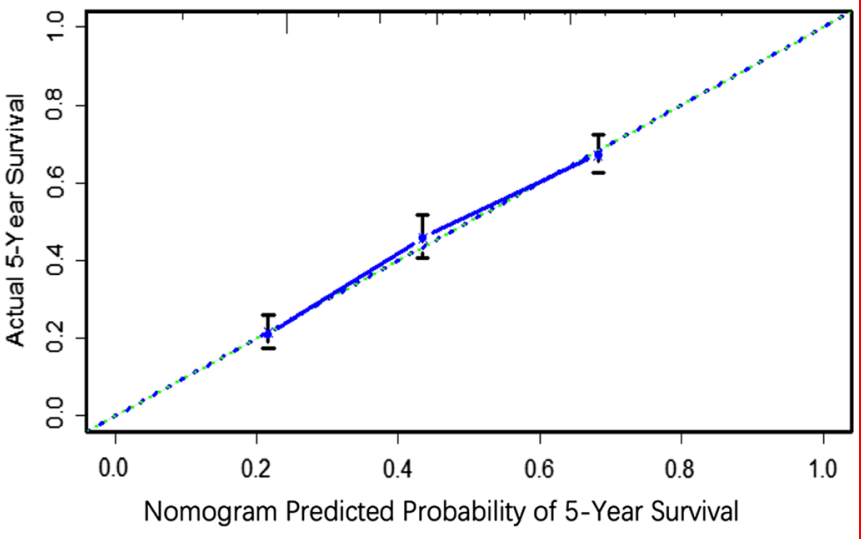

Fig. 4 A was a nomogram which was composed of the independent prognostic factors to predict the 5-year overall survival rate of gastric cancer patients, while B showed a calibration curve produced in the independent cohort after applying this nomogram. The risk value of 5-year overall survival rate is calculated by drawing a vertical line to the point on the axis for each of the factors. The points for each factor are summed and located on the total point line. And thereafter, the bottom line corresponding vertically to the above total line demonstrates the individual predictive value for 5-year overall survival rate

to be with a better survival rate than those who were with Fib-high, in both the cohorts before and after matching (Fig. 3c, d).

Cox's regression model is displayed in Table 3 to analyze the prognostic value of fibrinogen in the propensityscore-matched cohort. Univariate analysis showed that some clinicopathologic features, including of gender $(\mathrm{HR}=0.843, p=0.035)$, age $(\mathrm{HR}=1.404, p=0.009)$, tumor size $(\mathrm{HR}=1.051, p=0.037)$, fibrinogen $(\mathrm{HR}=$ $1.109, p=0.011)$, tumor differentiation $(\mathrm{HR}=1.012$, $p=0.023)$, macroscopic type $(\mathrm{HR}=0.893, p=0.017), \mathrm{T}$ stage $(\mathrm{T} 2$ vs. $\mathrm{T} 1, \mathrm{HR}=1.739, p=0.005$; $\mathrm{T} 3$ vs. $\mathrm{T} 1$, $\mathrm{HR}=2.031, \quad p=0.003 ; \quad \mathrm{T} 4 \quad$ vs. $\mathrm{T} 1, \quad \mathrm{HR}=2.894$, $p<0.001$ ), $\mathrm{N}$ stage (N1 vs. N0, HR $=1.735, p=0.011$; $\mathrm{N} 2$ vs. N0, HR $=2.214, p=0.009 ; \mathrm{N} 3$ vs. N0, HR = $2.975, p<0.001)$ and chemotherapy status $(\mathrm{HR}=1.156$, $p=0.035$ ), were significant prognostic factors, whereas tumor location(HR $=0.906, p=0.081$ ), surgical approach $(\mathrm{HR}=0.989, p=0.078)$, and gastrectomy extent (Distal vs. Total gastrectomy, $\mathrm{HR}=0.877, p=0.069$; Proximal vs. Total gastrectomy, $\mathrm{HR}=1.125, p=0.103$ ) were illustrated to be not significant risk factors for overall survival. However, multivariate Cox regression analysis indicated that only fibrinogen and pathological $\mathrm{T}$ and $\mathrm{N}$ stages were significantly associated with patients' survival prognosis, revealing that they were independent prognostic factors.

Nomogram was performed in this study to predict 5-year overall survival (Fig. 4a). Independent prognostic factors, fibrinogen and $\mathrm{T}$ stage and $\mathrm{N}$ stages, were included in the nomogram plot, either in the inner training cohort or in the validation cohort. Kaplan-Meier survival analysis for fibrinogen were suggested in Fig. 5, and the corresponding calibration curves in the training and validation cohorts showed that the predictive probability was closely similar to the actual 5-year overall survival rate. Besides, this nomogram was applied in another independent cohort to validate its prognostic accuracy in Fig. $4 \mathrm{~b}$, the calibration curve showed a similar result as illustrated in the validation cohort.

Furthermore, AIC value and c-index were utilized to compare the discriminatory ability and prognostic accuracy, respectively, between this nomogram and TNM staging system in Table 4. Significant improvement in prognostic prediction was found not only in the training and validation cohort, but also in the independent cohort. To be specific, TNM staging system was demonstrated to produce a smaller c-index (all $p<0.05)$ and a larger AIC (all $p<0.05$ ) than the present nomogram.

\section{Discussion}

Fibrinogen was identified to be an independent prognostic factor for gastric cancer patients, and additional attention should be paid on it. No uniform consensus, nonetheless, has been reached yet so far, due to the evaluation criteria and because of limitation of inconsistent cutoff point [8, 13-16]. Actually, no well-recognized and unified cutoff point for fibrinogen existed for gastric cancer. We applied the optimal cutoff point, $3.20 \mathrm{~g} / \mathrm{L}$, to categorize patients into Fib-high and Fib-low groups in this study, which indicated more predictive accuracy and showed a better discriminatory ability than those reported previously. Moreover, we found that patients in Fib-high group were 

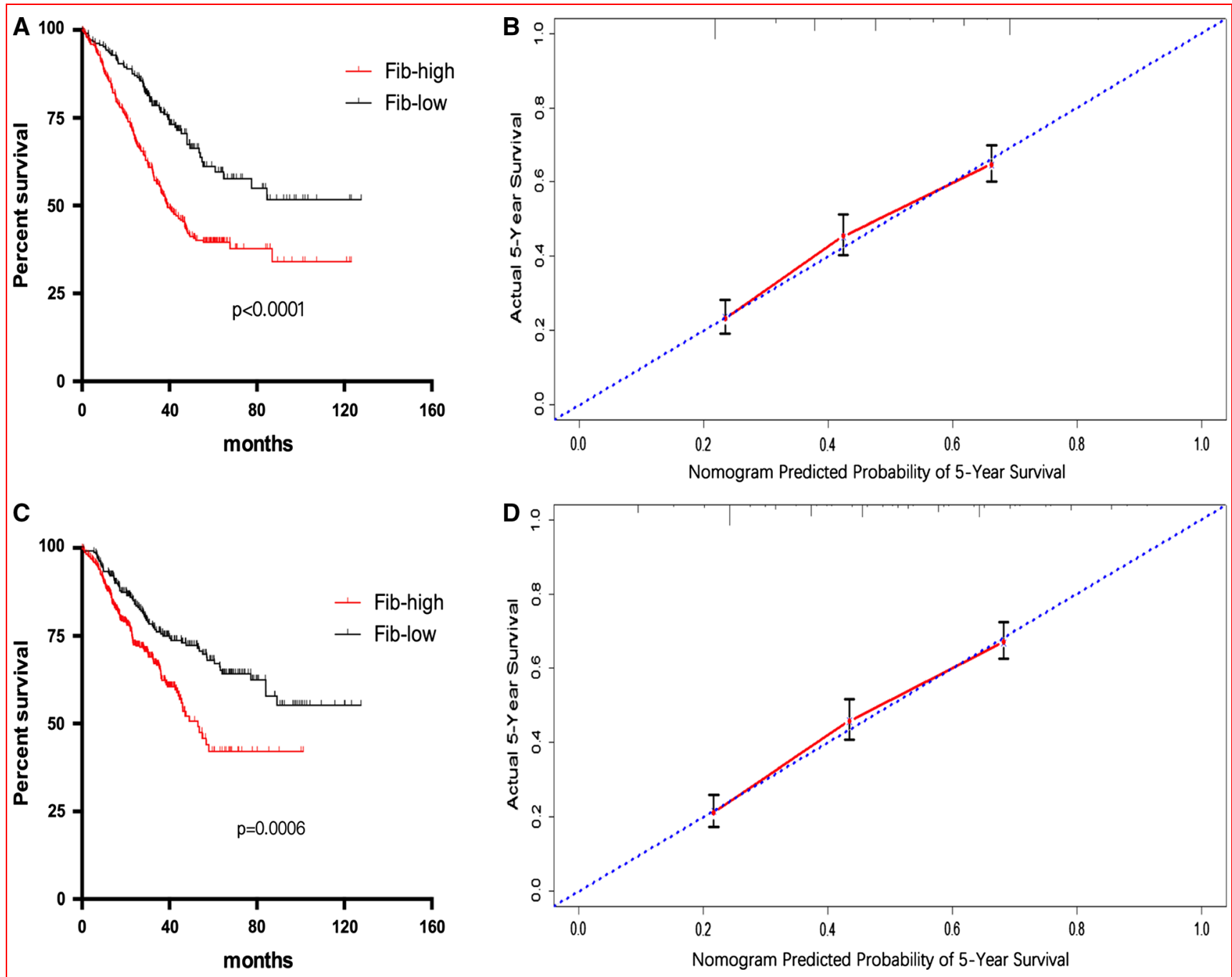

Fig. 5 Kaplan-Meier survival curves of fibrinogen for patients in the training (a) and validation cohort (c), and the calibration curves produced by the nomogram in the training (b) and validation cohort (d), respectively. The significance of difference between survival curves was calculated by the log-rank test

Table 4 The comparison of accuracy and discriminatory ability between the present nomogram and TNM staging system

\begin{tabular}{|c|c|c|c|c|c|c|c|c|c|}
\hline \multirow[t]{2}{*}{ Factor } & \multicolumn{3}{|c|}{ Training cohort } & \multicolumn{3}{|c|}{ Validation cohort } & \multicolumn{3}{|c|}{ Independent cohort } \\
\hline & TNM & Nomogram & $P$ value & TNM & Nomogram & $P$ value & TNM & Nomogram & $P$ value \\
\hline C-index & 0.682 & 0.723 & 0.003 & 0.702 & 0.734 & $<0.001$ & 0.691 & 0.719 & $<0.001$ \\
\hline AIC & 780.4 & 730.1 & 0.046 & 832.2 & 798.4 & 0.035 & 965.6 & 872.7 & 0.032 \\
\hline
\end{tabular}

AIC Akaike information criterion

companied with more aggressive features and worse biological behavior than patients in Fib-low group, both in the training and in the validation cohort, which illustrated that fibrinogen might be correlated with the tumor progression of gastric cancer.

Specifically, patients in Fib-high group was found more frequently with the presence of deep tumor invasion and advanced macroscopic type as well as wide lymph nodes metastasis, being consistent with previous studies in which a strong correlation between fibrinogen and increased metastatic potential, advanced tumor stage in various tumors was increasingly being recognized [7, 8, 13-16]. Fibrinogen, as an acute-phase protein regulating clotting and fibrinolysis in the blood, could probably be induced by cancer cells directly activating the clotting response or indirectly stimulating mononuclear cells secretion [22], 
which could be the reason why patients with aggressive features were more frequently distributed in the Fib-high group in the present study. However, we found that histology type and tumor size were not consistently allocated in two groups, as for the training and validation cohort. This inconformity of these two variables above might be attributed to their uneven distributions of the two cohorts. Meanwhile, our results demonstrated that there might exist multicollinearity with fibrinogen, and these could be the potential confounding factors for fibrinogen. Therefore, propensity score matching was applied to reduce the multicollinearity and to balance the baseline. After matching, fibrinogen was illustrated to be an independent predictor, and patients with Fib-low showed a better survival as compared to those with Fib-high.

In addition to $\mathrm{T}$ and $\mathrm{N}$ stages, fibrinogen was also demonstrated to be an independent predictor by multivariate Cox regression analysis. A nomogram including of these three independent predictors was constructed in the training cohort, and calibration curves were further performed to validate this nomogram not only in the inner validation cohort but also in the independent cohort, which revealed a reliability and a good concordance estimate for the 5-year overall survival. Moreover, an excellent staging system is expected to provide guidance of choosing appropriate adjuvant treatment and accurate prognostic estimation [23]. Hence, AIC value and C-index were used to demonstrate how much improvement was achieved in prognostic prediction using this nomogram. The present nomogram, as compared with TNM staging system, showed a better discriminatory ability and indicated more predictive accuracy in survival prediction, not only in training cohort and validation cohort but also in the independent cohort.

Apart from our clinical findings that preoperative plasma fibrinogen was correlated with aggressive clinicopathologic features, tumor progression and overall survival of gastric cancer patients, previous in vitro studies revealed that fibrinogen can enhance the cell migration and invasion of gallbladder cancer [24] and esophageal squamous cell carcinoma [7] by inducing epithelial-mesenchymal transition (EMT) process through the signal pathway of p-AKT/p-mTOR, which demonstrated that fibrinogen promoted cell proliferation in vitro, reminding us that fibrinogen could stimulate the cancer cells' growth via a certain kind of pathway which needed further study to elucidate its mechanism.

Limitations also existed in our study. Firstly, some factors could be observed by chance regardless of the large number of patients, and optimal cutoff point of preoperative serum fibrinogen could possibly make sense only in our study. In addition, only fibrinogen among the factors entered in the nomogram could be obtained preoperatively, which could limit its application in the formulation of optimal therapeutic strategy. Moreover, the mechanism of fibrinogen enhancing the proliferation of gastric cancer cells was still unknown. Therefore, more investigations are needed to evaluate the significance of preoperative plasma fibrinogen and to elucidate the mechanism affecting proliferation before stronger statement can be done.

\section{Conclusion}

Preoperative plasma fibrinogen levels in gastric cancer patients were significantly correlated with tumor progression, which could be regarded as a reliable marker for the prognostic prediction, and nomogram based on fibrinogen may improve the prognostic accuracy for survival prediction.

Acknowledgements The authors Lin-Yong Zhao, Yong-Liang Zhao, and Juan-Jiang Wang contributed equally in this paper. This study was funded by (1) 1.3 .5 project for disciplines of excellence, West China Hospital, Sichuan University, No. ZY2017304; (2) Foundation of Science and Technology Department of Sichuan Province, No.2018SZ0147; (3) China Postdoctoral Science Foundation, Nos. 2018M633374, 2016T90853; (4) Post-Doctor Research Project, West China Hospital, Sichuan University, 2018HXBH013.

\section{Compliance with ethical standards}

Conflict of interest The authors declare no potential conflicts of interest.

Ethics approval and consent to participate Patient records from three medical centers were de-identified and anonymized prior to analysis. The Research Ethics Committee of West China Hospital and Surgical Gastric Cancer Patient Registry approved this retrospective study (No. WCH-SGCPR-2017-08).

Informed consent As a retrospective analysis, informed consent in this study was waived.

Open Access This article is distributed under the terms of the Creative Commons Attribution 4.0 International License (http://crea tivecommons.org/licenses/by/4.0/), which permits unrestricted use, distribution, and reproduction in any medium, provided you give appropriate credit to the original author(s) and the source, provide a link to the Creative Commons license, and indicate if changes were made.

\section{References}

1. Chen W, Zheng R, Baade PD, et al (2016) Cancer statistics in China, 2015. CA 66(2):115-132. https://doi.org/10.3322/caac. 21338

2. Lip GY, Chin BS, Blann AD (2002) Cancer and the prothrombotic state. Lancet Oncol 3(1):27-34

3. Goldenberg N, Kahn SR, Solymoss S (2003) Markers of coagulation and angiogenesis in cancer-associated venous 
thromboembolism. J Clin Oncol 21(22):4194-4199. https://doi. org/10.1200/jco.2003.05.165

4. Palumbo JS, Kombrinck KW, Drew AF et al (2000) Fibrinogen is an important determinant of the metastatic potential of circulating tumor cells. Blood 96(10):3302-3309

5. Palumbo JS, Talmage KE, Massari JV et al (2005) Platelets and fibrin(ogen) increase metastatic potential by impeding natural killer cell-mediated elimination of tumor cells. Blood 105(1):178-185. https://doi.org/10.1182/blood-2004-06-2272

6. Simpson-Haidaris PJ, Rybarczyk B (2001) Tumors and fibrinogen. The role of fibrinogen as an extracellular matrix protein. Ann N Y Acad Sci 936:406-425

7. Zhang F, Wang Y, Sun P et al (2017) Fibrinogen promotes malignant biological tumor behavior involving epithelial-mesenchymal transition via the p-AKT/p-mTOR pathway in esophageal squamous cell carcinoma. J Cancer Res Clin Oncol. https://doi.org/10.1007/s00432-017-2493-4

8. Yu X, Hu F, Yao Q et al (2016) Serum fibrinogen levels are positively correlated with advanced tumor stage and poor survival in patients with gastric cancer undergoing gastrectomy: a large cohort retrospective study. BMC Cancer 16:480. https://doi.org/ 10.1186/s12885-016-2510-z

9. Troppan KT, Melchardt T, Wenzl K et al (2016) The clinical significance of fibrinogen plasma levels in patients with diffuse large B cell lymphoma. J Clin Pathol 69(4):326-330. https://doi. org/10.1136/jclinpath-2015-203356

10. Krenn-Pilko S, Langsenlehner U, Stojakovic T et al (2015) An elevated preoperative plasma fibrinogen level is associated with poor disease-specific and overall survival in breast cancer patients. Breast (Edinburgh, Scotland) 24(5):667-672. https://doi. org/10.1016/j.breast.2015.08.003

11. Wakatsuki K, Matsumoto S, Migita K et al (2017) Preoperative plasma fibrinogen is associated with lymph node metastasis and predicts prognosis in resectable esophageal cancer. World J Surg 41(8):2068-2077. https://doi.org/10.1007/s00268-017-3991-x

12. Caliskan S, Sungur M (2017) Fibrinogen and D-dimer levels in prostate cancer: preliminary results. Prostate Int 5(3):110-112. https://doi.org/10.1016/j.prnil.2017.05.001

13. Yu W, Wang Y, Shen B (2016) An elevated preoperative plasma fibrinogen level is associated with poor overall survival in Chinese gastric cancer patients. Cancer Epidemiol 42:39-45. https:// doi.org/10.1016/j.canep.2016.03.004

14. Arigami T, Uenosono Y, Ishigami S et al (2016) A novel scoring system based on fibrinogen and the neutrophil-lymphocyte ratio as a predictor of chemotherapy response and prognosis in patients with advanced gastric cancer. Oncology 90(4):186-192. https:// doi.org/10.1159/000444494
15. Lee SE, Lee JH, Ryu KW et al (2012) Preoperative plasma fibrinogen level is a useful predictor of adjacent organ involvement in patients with advanced gastric cancer. J Gastric Cancer 12(2):81-87. https://doi.org/10.5230/jgc.2012.12.2.81

16. Yamashita H, Kitayama J, Kanno N et al (2006) Hyperfibrinogenemia is associated with lymphatic as well as hematogenous metastasis and worse clinical outcome in T2 gastric cancer. BMC Cancer 6:147. https://doi.org/10.1186/1471-2407-6-147

17. Washington K (2010) 7th edition of the AJCC cancer staging manual: stomach. Ann Surg Oncol 17(12):3077-3079. https://doi. org/10.1245/s10434-010-1362-z

18. Camp RL, Dolled-Filhart M, Rimm DL (2004) X-tile: a new bioinformatics tool for biomarker assessment and outcome-based cut-point optimization. Clin Cancer Res 10(21):7252-7259. https://doi.org/10.1158/1078-0432.ccr-04-0713

19. Kulkarni GS, Hermanns T, Wei Y et al (2017) Propensity score analysis of radical cystectomy versus bladder-sparing trimodal therapy in the setting of a multidisciplinary bladder cancer clinic. J Clin Oncol 35(20):2299-2305. https://doi.org/10.1200/jco.2016. 69.2327

20. Huitzil-Melendez FD, Capanu M, O'Reilly EM et al (2010) Advanced hepatocellular carcinoma: which staging systems best predict prognosis? J Clin Oncol 28(17):2889-2895. https://doi. org/10.1200/jco.2009.25.9895

21. Zhao LY, Li CC, Jia LY et al (2016) Superiority of lymph node ratio-based staging system for prognostic prediction in 2575 patients with gastric cancer: validation analysis in a large single center. Oncotarget 7(32):51069-51081. https://doi.org/10.18632/ oncotarget.9714

22. Hillen HF (2000) Thrombosis in cancer patients. Ann Oncol 11(Suppl 3):273-276

23. Rice TW, Rusch VW, Ishwaran H et al (2010) Cancer of the esophagus and esophagogastric junction: data-driven staging for the seventh edition of the American Joint Committee on Cancer/ International Union Against Cancer Cancer Staging Manuals. Cancer 116(16):3763-3773. https://doi.org/10.1002/cncr.25146

24. Shu YJ, Weng H, Bao RF et al (2014) Clinical and prognostic significance of preoperative plasma hyperfibrinogenemia in gallbladder cancer patients following surgical resection: a retrospective and in vitro study. BMC Cancer 14:566. https://doi.org/ 10.1186/1471-2407-14-566

Publisher's Note Springer Nature remains neutral with regard to jurisdictional claims in published maps and institutional affiliations. 